\title{
Study on Application of Grey Prediction Model in Superalloy MAR-247 Machining
}

\author{
Chen Shao-Hsien \\ Department of Mechanical Engineering, National Chin-Yi University of Technology, No. 57, Section 2, \\ Zhongshan Road, Taiping District, Taichung 41170, Taiwan
}

Correspondence should be addressed to Chen Shao-Hsien; e6036@ncut.edu.tw

Received 27 February 2015; Accepted 23 June 2015

Academic Editor: Pavel Lejcek

Copyright (C) 2015 Chen Shao-Hsien. This is an open access article distributed under the Creative Commons Attribution License, which permits unrestricted use, distribution, and reproduction in any medium, provided the original work is properly cited.

Superalloy MAR-247 is mainly applied in the space industry and die industry. With its characteristics of mechanical property, fatigue resistance, and high temperature corrosion resistance, therefore, it is mainly applied in machine parts of high temperature and corrosion resistance, such as turbine blades and rotor of the aeroengine and turbine assembly in the nuclear power plant. However, considering that its properties of high strength, low thermal conductivity, being difficult to soften, and work hardening may reduce the life of cutting-tool and weaken the surface accuracy, the study provided minimizing experiment occurring during milling process for superalloy material. As a statistical approach used to analyse experiment data, this study used GM(1,1) in the grey prediction model to conduct simulation and then predict and analyze its characteristics based on the experimental data, focusing on the tool life and surface accuracy. Moreover, with the superalloy machining parameters of the current effective application improved grey prediction model, it can decrease the errors, extend the tool life, and improve the prediction precision of surface accuracy.

\section{Introduction}

In the milling progress, the nickel-base alloy may shorten the tool life and it's surface accuracy to bad due to it's material characteristics, which are properties of hard-to-cut materials in the milling process currently, and the reasons for being hard-to-cut are listed in the following:

(1) Work hardening ability: when processing the nickelbase materials, the phenomenon of the precipitation hardening appearing to the material may result in difficulty in machining and finally cause cutting-tool flaking and severe wear at the blade end, so that the surface accuracy is degraded.

(2) Low thermal conductivity: for common steel cutting, though cutting heat may arise, it is generally brought away by the cuttings to some degree; on the contrary, the superalloy, due to its low thermal conductivity, is easy to accumulate the cutting heat of the cuttingtool and work-piece; and its high yield point and tensile strength, large cutting impedance, and blade end of the tool are easy to cause high pressure, high temperature, and plastic deformation.

(3) The affinity between tool and superalloy: in the cases of discontinuous cutting like milling, phenomenon of welding may occur at the tool end or in the cuttings, while the melted objects may tumble in the workpieces, which will cause a larger impact force and even lead to the cutting-tool flaking [1-4].

This study use of $\operatorname{GM}(1,1)$ in the grey prediction model to simulate and predict the correlations between tool wear and surface accuracy, particularly the significant effects of superalloy MAR-247 on the tool wear. The $\operatorname{GM}(1,1)$ prediction model in grey system theory is characterized by the fact that it can conduct prediction with a small amount of cutting data and experimental data; however, the $\operatorname{GM}(1,1)$ model is the convergent form of first-order linear differential equation, so the repeated AGO operations may increase the errors. In the study, it used the exponential smoothing to make improvements, so as to realize the goal of reducing the prediction errors of tool wear and surface accuracy. 


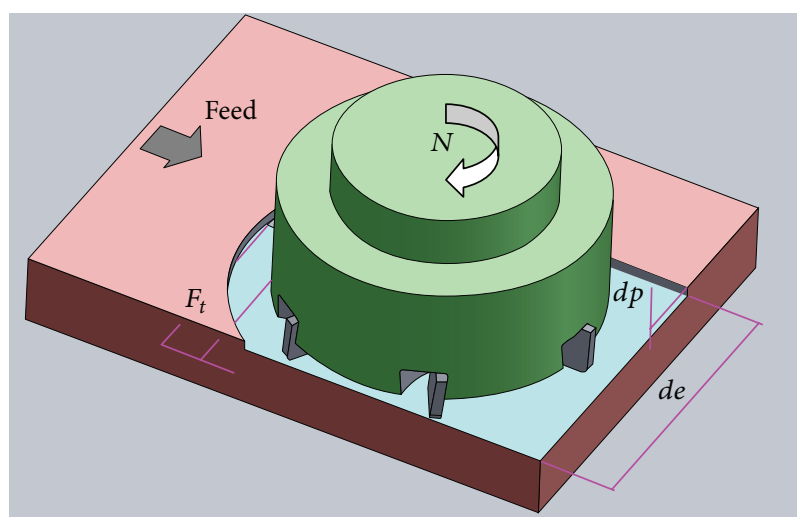

FIGURE 1: Diagram of cutting parameter.

\section{Cutting Principle and Material Properties}

2.1. Cutting Principle. The major impact factors in cutting include CNC precision machine, tool material, machining parameters, and controller. In the study, machining parameter is selected as the variable factor, including the depth of cut (DOC) and feeding speed; the fixed factors cover the rotation speed, cutting compound, and so forth. Figure 1 is the diagram of cutting parameter, in which the object function refers to the life and surface accuracy, with the general formula of cutting principle shown in the following.

Spindle speed (r.p.m.) is calculated based on the different tool diameter and different work-piece cutting speed.

The tool diameter is inversely proportional to the revolution speed of main shaft, while the cutting speed is directly proportional to the revolution speed, known from the following equation:

$$
N=\frac{1000 V c}{\pi D}(\mathrm{rpm})
$$

$V c$ is cutting speed $(\mathrm{m} / \mathrm{mim})$ and $D$ is tool diameter $(\mathrm{mm})$.

Cutting feed rate is one of the factors influencing the cutting time; that is, the faster the cutting feed rate produces, the shorter the cutting time is. Besides, the material removal rate is also improved, which is reduced on the contrary. The calculation formula of cutting feed rate is shown in the following:

$$
F=N \times F_{t} \times t
$$

$N$ is revolution speed; $F_{t}$ is the cutting output of each tooth $(\mathrm{mm} /$ tooth $) ; t$ is tooth number.

2.2. Metal Properties of Supperalloy MAR-M247. Generally, the nickel-base alloy will generate FCC matrix ( $\gamma$ phase) after the aging treatment to separate out the discoid $\gamma^{\prime \prime}(\mathrm{Ni3Nb})$ phase and finally turn out the integrated response; however, MAR-M247 fails to produce precipitation significantly but mainly separate out the $\gamma^{\prime}\left[\mathrm{Ni}_{3}(\mathrm{Ti}, \mathrm{Al})\right]$ phase with tiny spherical precipitated phase. The mechanical properties of alloy like strength and hardness grow as the size of $\gamma^{\prime \prime}$ particle increases. The quantity of generated $\gamma^{\prime}$ may affect MAR-247's performance of heat-resistance; namely, the content is higher and the high temperature strength is better. Therefore, $\mathrm{Al}, \mathrm{Ti}$, $\mathrm{Nb}$, and Ta dissolved in the nickel-base matrix all contribute to the generation of $\gamma^{\prime}$ phase. If the amount of $\gamma^{\prime \prime}$ and $\gamma^{\prime}$ phase precipitation is enough, the alloy can achieve the highest strength, which is also the reason why the alloy performs even much higher strength under high temperature. Meanwhile, its processability becomes worse. The impacts of temperature on material changes are shown in Figure 2.

In addition, carbon element also plays a crucial influence on the mechanical properties of superalloy MAR-M247, since the carbon itself cannot form the carbide with nickel but bonding with other alloy elements. Commonly, there are there types of precipitations $\left(\mathrm{MC}, \mathrm{M}_{6} \mathrm{C}\right.$, and $\mathrm{M}_{23} \mathrm{C}_{6}$ ) generated from nickel-base alloy and carbide. The studies reveal that $\mathrm{MC}$ (high temperature tungsten carbide phase) is separated out in the form of lump, which has little help to the materials. However, $\mathrm{M}_{6} \mathrm{C}$ is often precipitated in grain boundary in form of lumps, so the size of particle can be controlled. It is necessary to desalinate the carbide in the material to avoid the $\mathrm{M}_{7} \mathrm{C}_{3}$ in the crystal grain generated by $\mathrm{TiC}[6]$.

\section{Construction of Prediction Model}

3.1. Grey Prediction $\operatorname{GM}(1,1)$ Model. The grey prediction model is primary $\operatorname{GM}(1,1)$ developed by Deng [7], mainly applied under conditions of less model information, more impact variables, and uncertain behavior models, and the operation model includes the works of correlation analysis, model construction, prediction, and control [6].

The grey prediction model GM $(n, h)$ is a dynamic model, in which $n$ refers to the order of differential equation and $h$ the number of variables of the differential equation, so $\operatorname{GM}(1,1)$ is the first-order differential equation [8]. It mainly adopts the training value to conduct mode- 1 which uses a few training values to finish the prediction; the current prediction procedure of $\operatorname{GM}(1,1)$ is described in the following $[9,10]$.

Step 1 (primitive sequence and experimental values). Consider the following:

$$
x^{(0)}=\left(x^{(0)}(1), x^{(0)}(2), x^{(0)}(3), \ldots, x^{(0)}(n)\right),
$$

$n \geq 2$.

Step 2 (conducting the Accumulated Generating Operation (AGO)). Let $x^{(1)}$ be the transformation sequence of $x^{(0)}$ at $T$ :

$$
\begin{aligned}
x^{(1)} & =\left(x^{(1)}(1), x^{(1)}(2), x^{(1)}(3), \ldots, x^{(1)}(n)\right) \\
x^{(1)}(k) & =\sum_{m=1}^{k} x^{(0)}(m) .
\end{aligned}
$$

Consequently, model of the first-order differential equation $\mathrm{GM}(1,1)$ is

$$
\frac{d x^{(1)}}{d t}+a x^{(1)}=b,
$$




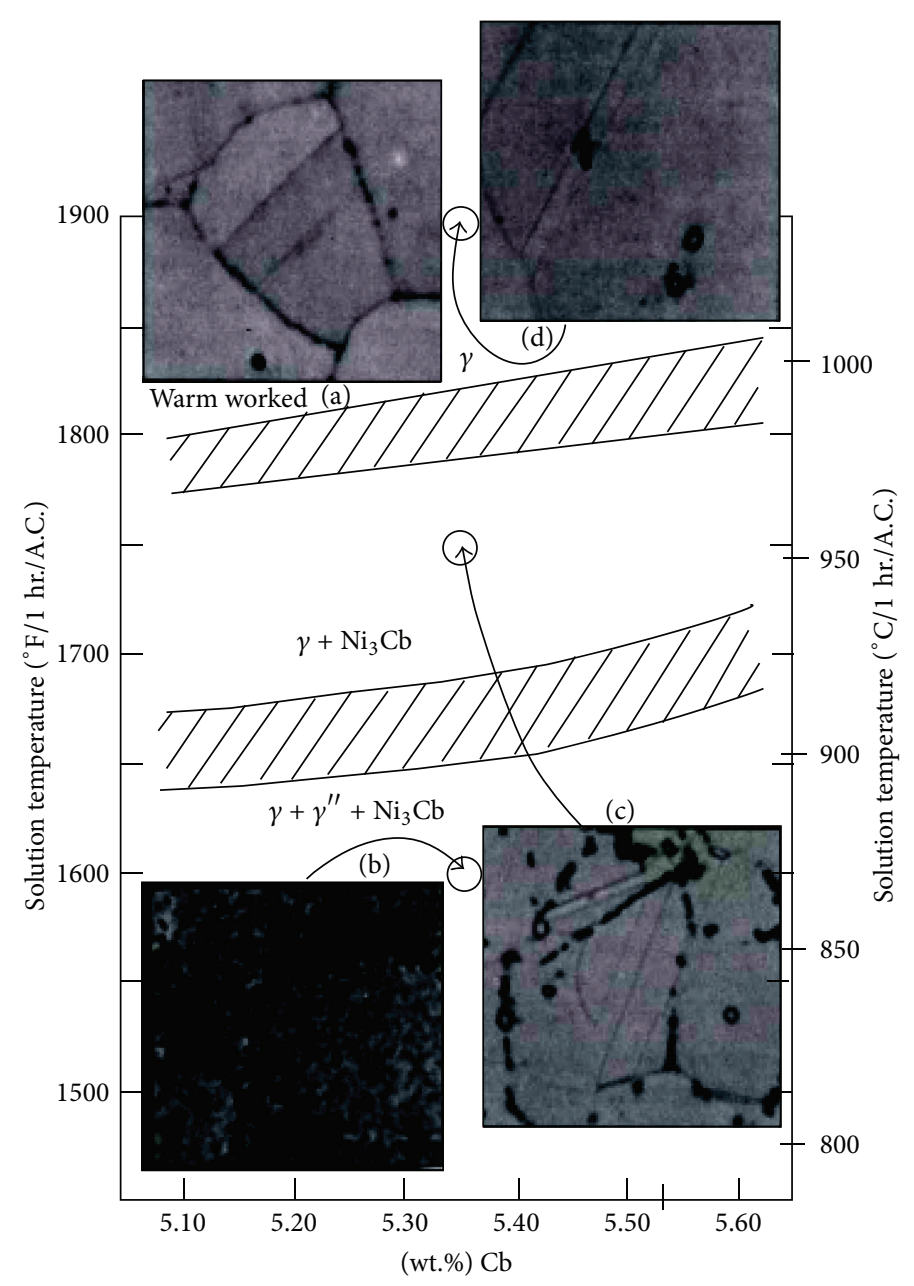

FIGURE 2: Influences of temperature change on the material structure [5].

wherein $t$ is independent variable, $a$ is the development coefficient, and $b$ is the grey control variable.

Step 3 (conversion treatment of the former and latter terms). Consider the following:

$$
\frac{d x^{(1)}}{d t} \longrightarrow x^{(1)}(k+1)-x^{(1)}(k)
$$

Through Inverse Accumulated Generating Operation (IAGO), it can be learned that

$$
x^{(1)}(k+1)-x^{(1)}(k)=x^{(0)}(k+1) .
$$

If $\alpha=0.5$, its definition in $x_{1}^{(1)}(t)$ would be

$$
x^{(1)}(k) \longrightarrow 0.5 x^{(1)}(k)+0.5 x^{(1)}(k-1)=z^{(1)}(k) .
$$

After collation, it is known that

$$
\frac{d x^{(1)}}{d t}+a x^{(1)}=b \longrightarrow x^{(0)}(k)+a z^{(1)}(k)=b
$$

Step 4 (determining $a, b$ by least square method). Consider the following:

$$
p=\left[\begin{array}{l}
a \\
b
\end{array}\right]=\left(B^{T} B\right)^{-1} B^{T} Y_{n}
$$

in which the accumulated matrix $B$ and constant term $Y_{n}$ are, respectively,

$$
B=\left[\begin{array}{ccc}
-z^{(1)}(2) & 1 \\
-z^{(1)}(3) & 1 \\
\vdots & & 1 \\
-z^{(1)}(n) & 1
\end{array}\right],
$$

$$
Y_{n}=\left[\begin{array}{c}
x(2) \\
x(3) \\
\vdots \\
x(n)
\end{array}\right] \text {. }
$$


Step 5 (constructing the grey prediction model). This equation in $\operatorname{GM}(1,1)$ is of

$$
\frac{d x^{(1)}}{d t}+a x^{(1)}=b
$$

wherein the primitive condition of $x^{(1)}$ is $x^{(0)}(1)=x^{(1)}(1)$.

According to the calculation method of ordinary differential equations (ODE), it can obtain the discretization response equation:

$$
\begin{aligned}
& \widehat{x}^{(1)}(k+1)=\left(x^{(0)}(1)-\frac{b}{a}\right) e^{-a(k-1)}+\frac{b}{a}, \quad k \geq R, \\
& \widehat{x}^{(1)}(k+1)=\left(x^{(0)}(1)\right) e^{-a k}+\frac{b}{a}\left(1-e^{-a k}\right), \quad k \geq 0 .
\end{aligned}
$$

Step 6. Process with Inverse Accumulated Generation Operation (IAGO). Consider the following:

$$
\widehat{x}^{(0)}(k+1)=\left(1-e^{a}\right)\left(x^{(0)}(1)-\frac{b}{a}\right) e^{-a k} .
$$

3.2. Improved $\operatorname{GM}(1,1)$ Model. Generally, the equations in $\operatorname{GM}(1,1)$ are the first-order differential equations, which all belong to convergence type, while the experimental value in this study is of progressive increase pattern which is of divergence type; thus it is modified into divergence form in the first-order differential equations and results in accumulated errors in IAGO. As a result, it is necessary to be converted and changed in the whitening formula. The following steps can be obtained after being modified $[11,12]$.

Step 1 (primitive sequence and experimental values). Consider the following:

$$
x^{(0)}=\left(x^{(0)}(1), x^{(0)}(2), x^{(0)}(3), \ldots, x^{(0)}(n)\right),
$$

$n \geq 2$.

Step 2 (executing the Accumulated Generating Operation $(\mathrm{AGO}))$. Let $x^{(1)}$ be the transformation sequence of $x^{(0)}$ at T:

$$
\begin{aligned}
x^{(1)} & =\left(x^{(1)}(1), x^{(1)}(2), x^{(1)}(3), \ldots, x^{(1)}(n)\right) \\
x^{(1)}(k) & =\sum_{m=1}^{k} x^{(0)}(m) .
\end{aligned}
$$

Consequently, model of the first-order differential equation $\operatorname{GM}(1,1)$ after modification is

$$
\frac{d x^{(1)}}{d t}-a x^{(1)}=b
$$

wherein $t$ is independent variable, $a$ the development coefficient, and $b$ the grey control variable.

Step 3 (conversion treatment of the former and latter terms). Consider the following:

$$
\frac{d x^{(1)}}{d t} \longrightarrow x^{(1)}(k+1)-x^{(1)}(k) .
$$

Through Inverse Accumulated Generating Operation (IAGO), it can be learned that

$$
x^{(1)}(k+1)-x^{(1)}(k)=x^{(0)}(k+1) .
$$

If $\alpha=0.5$, its definition in $x_{1}^{(1)}(t)$ would be

$$
x^{(1)}(k) \longrightarrow 0.5 x^{(1)}(k)+0.5 x^{(1)}(k-1)=z^{(1)}(k) .
$$

After collation, it is known that

$$
\frac{d x^{(1)}}{d t}-a x^{(1)}=b \longrightarrow x^{(0)}(k)-a z^{(1)}(k)=b .
$$

Step 4 (determining $a, b$ by least square method). Consider the following:

$$
p=\left[\begin{array}{l}
a \\
b
\end{array}\right]=\left(B^{T} B\right)^{-1} B^{T} Y_{n}
$$

in which the accumulated matrix $B$ and constant term $Y_{n}$ are, respectively,

$$
\begin{aligned}
B & =\left[\begin{array}{ccc}
-z^{(1)} & (2) & 1 \\
-z^{(1)} & (3) & 1 \\
\vdots & & 1 \\
-z^{(1)} & (n) & 1
\end{array}\right], \\
Y_{n} & =\left[\begin{array}{c}
x(2) \\
x(3) \\
\vdots \\
x(n)
\end{array}\right] .
\end{aligned}
$$

Step 5 (constructing the grey prediction model). The equation in $\operatorname{GM}(1,1)$ is of

$$
\frac{d x^{(1)}}{d t}-a x^{(1)}=b
$$

wherein the primitive condition of $x^{(1)}$ is $x^{(0)}(1)=x^{(1)}(1)$.

According to the solutions to ordinary differential equations (ODE), it can have obtain the discretization response equation:

$$
\widehat{x}^{(1)}(k+1)=\left(x^{(0)}(1)+\frac{b}{a}\right) e^{a(k-1)}-\frac{b}{a}, \quad k \geq N .
$$

Step 6. Process with Inverse Accumulated Generation Operation (IAGO). Consider the following:

$$
\widehat{x}^{(0)}(k+1)=\left(1-e^{-a}\right)\left(x^{(0)}(1)+\frac{b}{a}\right) e^{a(k-1)} .
$$

Step 7 (corrected value $u$ after improvement). It's mainly improves the error between the first point $x^{(0)}(1)$ of the primitive data and the first point $\widehat{x}^{(0)}(1)$ of the prediction value [11]:

$$
u=\frac{b}{a}-\left(x^{(0)}(1)+\frac{b}{a}\right) e^{-a}
$$




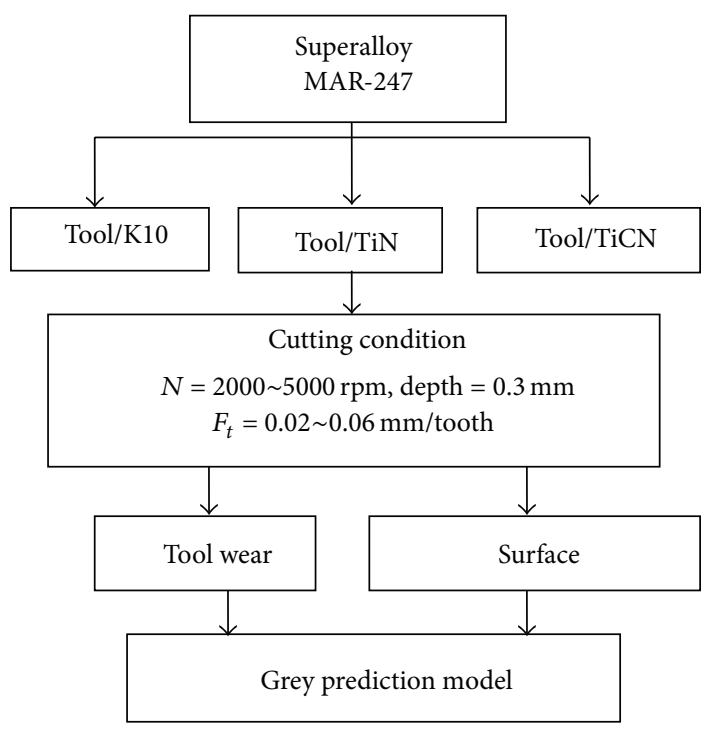

FIgURE 3: Experimental procedure chart.

The error amount $u(k)$ of the remaining points is determined as

$$
u(k)=e^{a(k-1)}\left(x^{(0)}(1)+u\right)-x^{(0)}(k) .
$$

Step 8 (training value after modification). Take $u(k)$ as the primitive data to get $u(k+1)$ by exponential smoothing, and its corrected value is $\bar{x}^{(0)}(k)$, shown as

$$
\bar{x}^{(0)}(k)=\widehat{x}^{(0)}(k)-u(k) \text {. }
$$

\section{Experimental Design and Facilities}

4.1. Experimental Facilities and Procedures. The study uses the machine centers to conduct the cutting experiment, with the highest revolution speed $N=6000 \mathrm{rpm}$, GOO feed rate $F=36 \mathrm{~m} / \mathrm{min}$, and the tool materials including tool $\mathrm{K} 10$ and coated TiN, TiCN tool K10. During the experiment, the author observes the relationship between tool wear and surface accuracy and utilizes the grey prediction model for analysis and prediction, which has realized the goal of practical application. The experimental procedure is displayed in Figure 3.

4.2. Experimental Analysis. Currently, the accuracy analysis is the criteria for evaluating the prediction methods, mainly based on the Mean Absolute Percentage Error (MAPE) and Root Mean Square Percentage Error (RMSE). The study is mainly used to evaluate and predict the tool life and surface accuracy, which then is coordinated with grey prediction $\operatorname{GM}(1,1)$ model and improved model to conduct prediction and discussion. The equations of MAPE and RMSE are as follows:

(1) Mean Absolute Percentage Error (MAPE):

$$
E_{\mathrm{MAPE}}=\frac{1}{n} \sum_{k=1}^{n} e_{k} ;
$$
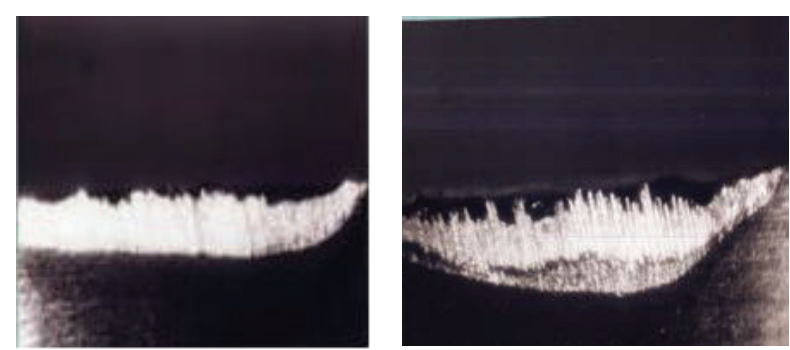

Figure 4: Tool wear of tool $\mathrm{K} 10(N=2000 \sim N=5000 \mathrm{rpm})$.

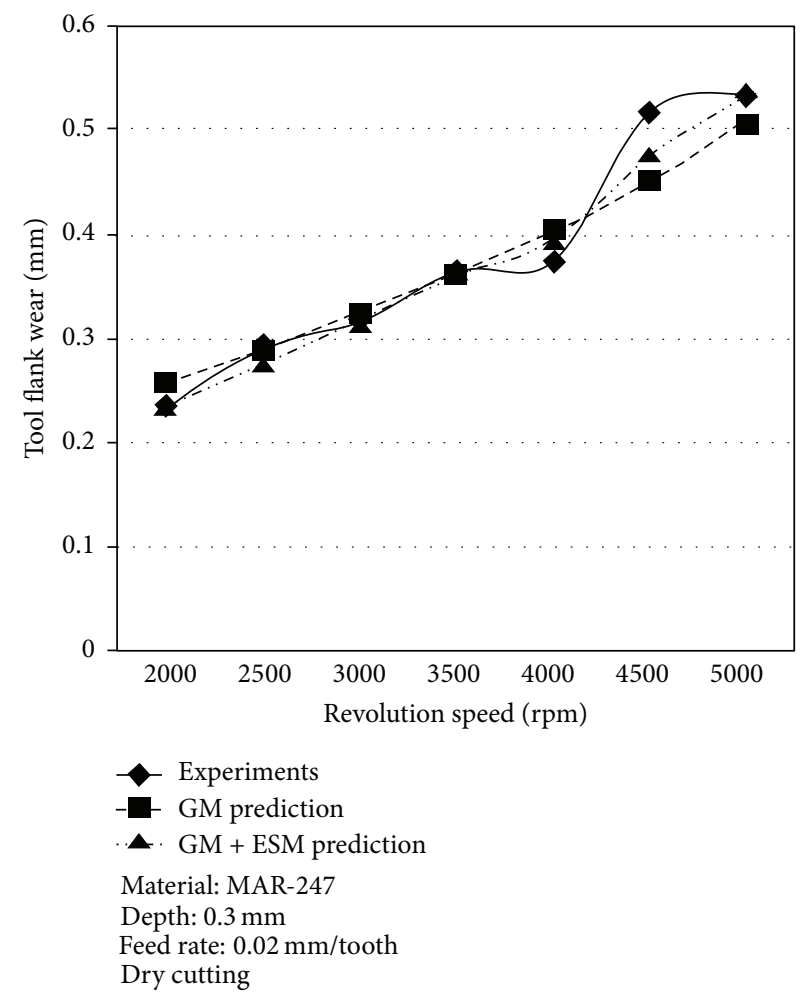

FIGURE 5: Relationships between revolution speed of main shaft and tool wear prediction.

(2) Root Mean Square Percentage Error (RMSE):

$$
E_{\mathrm{RMSP}}=\sqrt{\frac{1}{n} \sum_{k=1}^{n} e_{k}^{2}} .
$$

\section{Results and Discussion}

5.1. Impacts of Different Feed Rate on the Tool Wear. During the process of cutting nickel-base alloy, the material hardening and low heat conductivity may result in fast tool wear and degraded surface accuracy. Figure 4 shows the tool wear of tool K10, which reveals that a faster revolution speed leads to a severer tool wear. The study utilizes the grey prediction model and improved model to conduct analysis, showing that the higher revolution speed causes a severer tool wear. As shown in Figure 5, the improved model GM(1, 1) + MSE performs better than the conventional $\operatorname{GM}(1,1)$. From 
TABLE 1: Comparison sheet of the common and the improved grey prediction model.

\begin{tabular}{lccccc}
\hline \multicolumn{5}{c}{ Tool K10 } \\
$N(\mathrm{rpm})$ & Test & $\mathrm{GM}(1,1)$ & Error & $\mathrm{GM}(1,1)+\mathrm{MSE}$ & Error \\
\hline 2000 & 0.235 & 0.2577 & -0.0227 & 0.235 & 0 \\
2500 & 0.292 & 0.2883 & 0.0037 & 0.2775 & -0.0145 \\
3000 & 0.317 & 0.3225 & -0.0055 & 0.3159 & -0.0011 \\
3500 & 0.364 & 0.3608 & 0.0032 & 0.3608 & -0.0032 \\
4000 & 0.374 & 0.4036 & -0.0296 & 0.3933 & 0.0193 \\
4500 & 0.518 & 0.4514 & 0.0666 & 0.4768 & -0.0412 \\
5000 & 0.536 & 0.505 & 0.031 & 0.5393 & 0.0033 \\
MAPE & & & 0.0012 & & 0.0005 \\
RMSE & & & 0.0313 & & 0.0181 \\
\hline
\end{tabular}
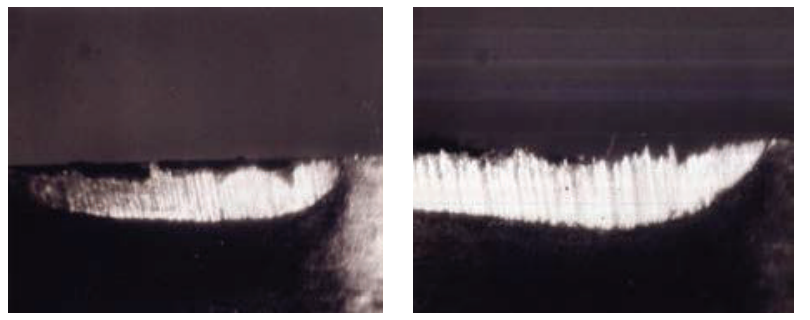

Figure 6: Tool wear of coated TiN tool $(N=2000 \sim N=$ $5000 \mathrm{rpm})$.

Table 1, the comparison sheet of common grey prediction model and improved grey prediction model, it can be learned that the error of MAPE is 0.0005 , smaller than 0.0012 , and error of RMSE is 0.0181 , smaller than 0.0313 . Therefore, if the primitive experimental values are increasing progressively, the improved grey prediction model $\operatorname{GM}(1,1)+$ MSE can reduce the errors.

When the coated TiN tool K10 is adopted, the observation of the tool wear shows that a higher revolution speed causes a severer tool wear, which is mainly because the numbers of tool frictions on site within the unit time make the temperature rise, making the material MAR-M247 harden and finally the tool is worn rapidly, as shown in Figures 6 and 7. In terms of the prediction model, the improved model $\operatorname{GM}(1,1)+$ MSE has a better performance than the common model $\operatorname{GM}(1,1)$. As listed in Table 2, the error of MAPE is reduced to 0.00043 after being improved, smaller than 0.00056, and that of RMSE to 0.0207, smaller than 0.0646 . As a result, if the primitive experimental value is increasing progressively, the improved grey prediction model $\operatorname{GM}(1,1)+$ MSE can reduce the error.

For coated TiCN tool K10, as shown in Table 3, the improved grey prediction model $\operatorname{GM}(1,1)+$ MSE can reduce the errors of MAPE and RMSE if the primitive experimental values are increasing progressively.

When the feed rate of each tooth is increased from $F_{t}=$ $0.02 \mathrm{~mm} /$ tooth to $F_{t}=0.06 \mathrm{~mm} /$ tooth, the tool wear is alleviated. As shown in Figure 8, higher revolution speed and feed rate play a more significant impact on the tool wear than the lower ones. The application of improved model
TABLE 2: Comparison sheet of the common and improved grey prediction model.

\begin{tabular}{lccccc}
\hline \multicolumn{5}{c}{ Tool K10+TiN } \\
$N(\mathrm{rpm})$ & Test & GM(1,1) & Error & GM(1,1) + MSE & Error \\
\hline 2000 & 0.171 & 0.2364 & -0.0654 & 0.171 & 0 \\
2500 & 0.264 & 0.2582 & 0.0058 & 0.2248 & -0.0392 \\
3000 & 0.272 & 0.282 & -0.01 & 0.264 & -0.008 \\
3500 & 0.313 & 0.308 & 0.005 & 0.307 & -0.006 \\
4000 & 0.415 & 0.3364 & 0.0786 & 0.3797 & -0.0353 \\
4500 & 0.455 & 0.3674 & 0.0876 & 0.4449 & -0.0101 \\
5000 & 0.506 & 0.4013 & 0.1047 & 0.509 & 0.003 \\
MAPE & & & 0.00561 & & 0.00043 \\
RMSE & & & 0.06464 & & 0.02068 \\
\hline
\end{tabular}

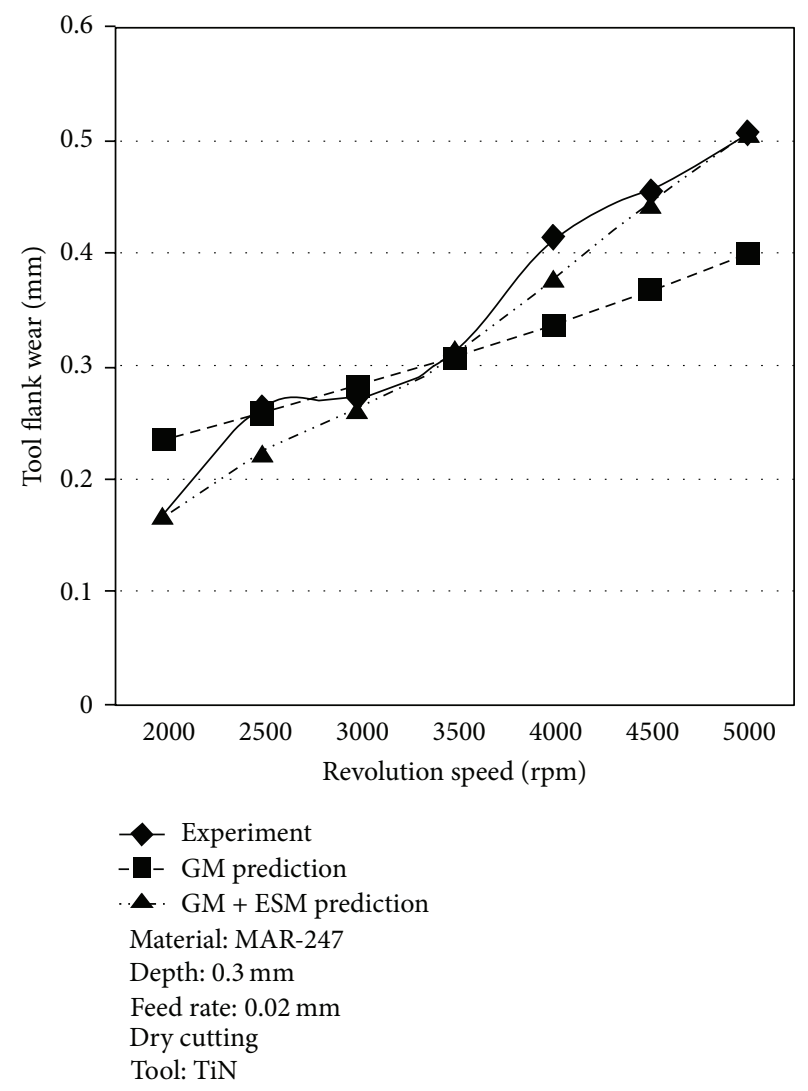

FIGURE 7: Relationships between revolution speed of main shaft and tool wear prediction.

$\operatorname{GM}(1,1)+$ MSE produces a better performance than model $\operatorname{GM}(1,1)$. From Figures 9 and 10, as well as the comparison sheet between the common and the improved grey prediction model, the errors can be reduced by adopting the improved model GM $(1,1)+$ MSE if the primitive experimental values are increasing progressively.

5.2. Impacts of Different Feed Rate on the Surface Accuracy. The severe tool wear may result in a worse surface roughness, especially for machining of nickel-base materials, and the tool wear is severer; while it is hard to observe the degree of tool 
TABLE 3: Comparison sheet of the common and improved grey prediction model.

\begin{tabular}{lccccc}
\hline \multicolumn{5}{c}{ Tool K10+TiCN } \\
$N(\mathrm{rpm})$ & Test & $\mathrm{GM}(1,1)$ & Error & $\mathrm{GM}(1,1)+\mathrm{MSE}$ & Error \\
\hline 2000 & 0.112 & 0.3929 & -0.2809 & 0.112 & 0 \\
2500 & 0.136 & 0.0947 & 0.0413 & 0.2306 & 0.0946 \\
3000 & 0.174 & 0.1299 & 0.0441 & 0.1631 & -0.0109 \\
3500 & 0.251 & 0.1782 & 0.0728 & 0.2277 & -0.0233 \\
4000 & 0.331 & 0.3352 & -0.0042 & 0.4044 & 0.0734 \\
4500 & 0.378 & 0.4597 & -0.0817 & 0.4162 & 0.0382 \\
5000 & 0.454 & 0.6305 & -0.1765 & 0.609 & 0.155 \\
MAPE & & & 0.06534 & & 0.02214 \\
RMSE & & & 0.134 & & 0.07605 \\
\hline
\end{tabular}

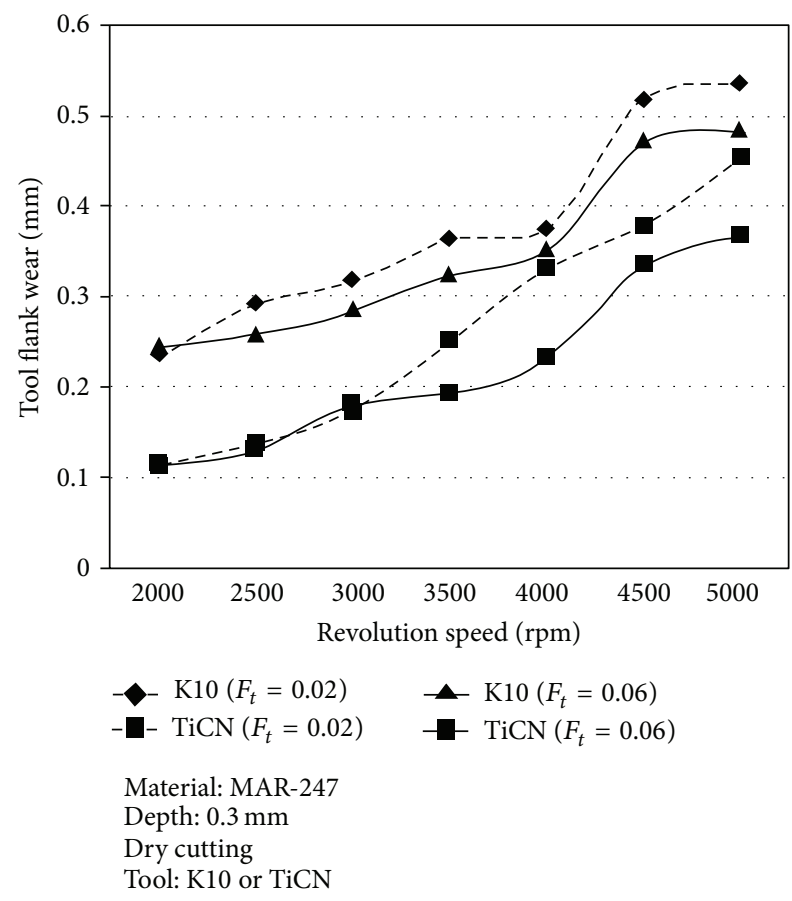

FIGURE 8: Relationships between revolution speed of main shaft and tool wear prediction.

wear and surface roughness online during cutting, generally, it only can get the relative data after finishing the cutting. The study uses the grey prediction principle to conduct analysis and prediction, finding that the tool wear is much more obvious when the revolution speed is higher, so that the surface roughness is accordingly degraded. From Figures 11 to 14 , the primary factors affecting the surface roughness include the radius of tool nose and feed rate of each tooth, which can be learned from the theoretical equation of cutting face roughness:

$$
R_{\max } \equiv \frac{F_{t}^{2}}{8 r_{n}} \times 1000(\mu \mathrm{m}) .
$$

As a result, the surface roughness is significantly affected by the tool wear rather than the radius of tool nose and
TABLE 4: Comparison sheet of the common and improved grey prediction model.

\begin{tabular}{lccccc}
\hline \multicolumn{5}{c}{ Tool/K10 } \\
$N(\mathrm{rpm})$ & Test & GM(1,1) & Error & GM + MSE & Error \\
\hline 2000 & 0.13 & 0.127 & 0.0029 & 0.13 & 0 \\
2500 & 0.13 & 0.132 & -0.0018 & 0.1326 & 0.0026 \\
3000 & 0.14 & 0.137 & 0.0034 & 0.1382 & -0.002 \\
3500 & 0.14 & 0.142 & -0.002 & 0.1416 & 0.0016 \\
4000 & 0.17 & 0.147 & 0.0232 & 0.1569 & -0.013 \\
4500 & 0.19 & 0.152 & 0.0378 & 0.1759 & -0.014 \\
5000 & 0.21 & 0.158 & 0.0522 & 0.1974 & -0.013 \\
MAPE & & & 0.0079 & & 0.002 \\
RMSE & & & 0.026 & & 0.008 \\
\hline
\end{tabular}

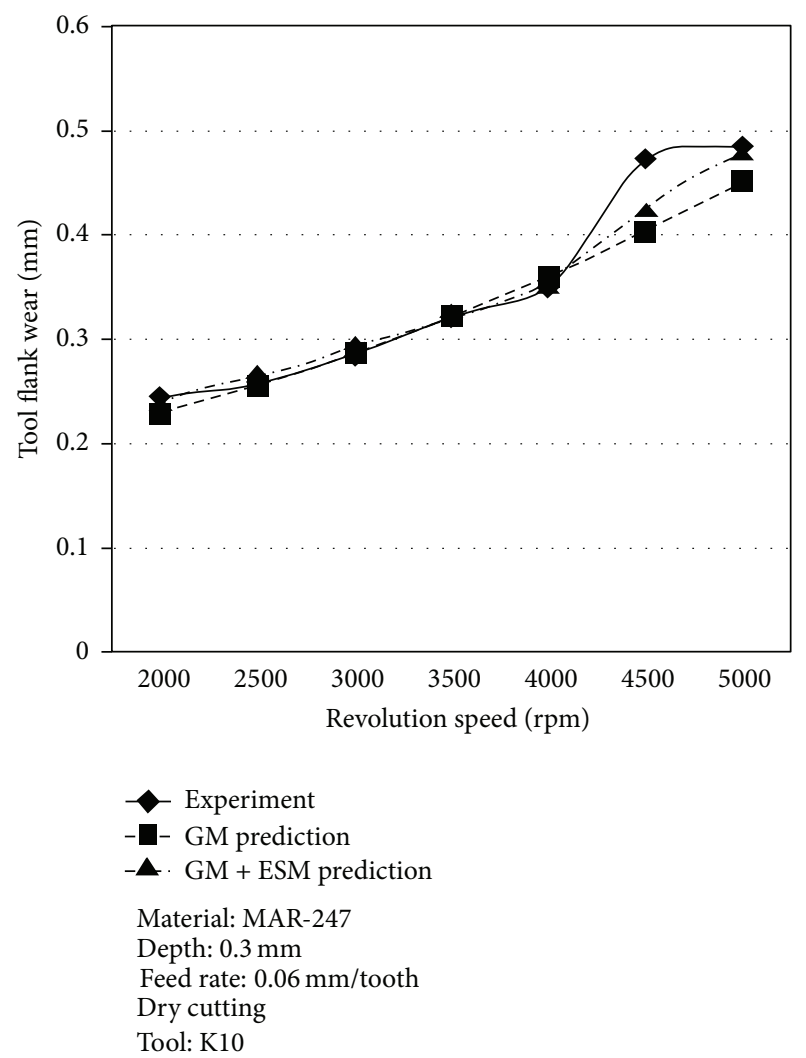

FIGURE 9: Relationships between revolution speed of main shaft and tool wear prediction.

feed rate of each tooth, particularly in high revolution speed regions. Among the prediction methods, the improved model $\operatorname{GM}(1,1)+$ MSE is superior to the common model $\operatorname{GM}(1,1)$. As listed in Table 4, the comparison sheet of the common and the improved grey prediction model, it can be learned that the error of MAPE is 0.002 after it is improved, smaller than 0.0079 , while that of RMSE is 0.0008 , smaller than 0.026 . Therefore, when the primitive experimental values are increasing progressively, the improved model $\operatorname{GM}(1,1)+$ MSE can reduce the errors.

When exploring the impacts of different tool material and feed rate on the surface roughness. It finds that the higher 


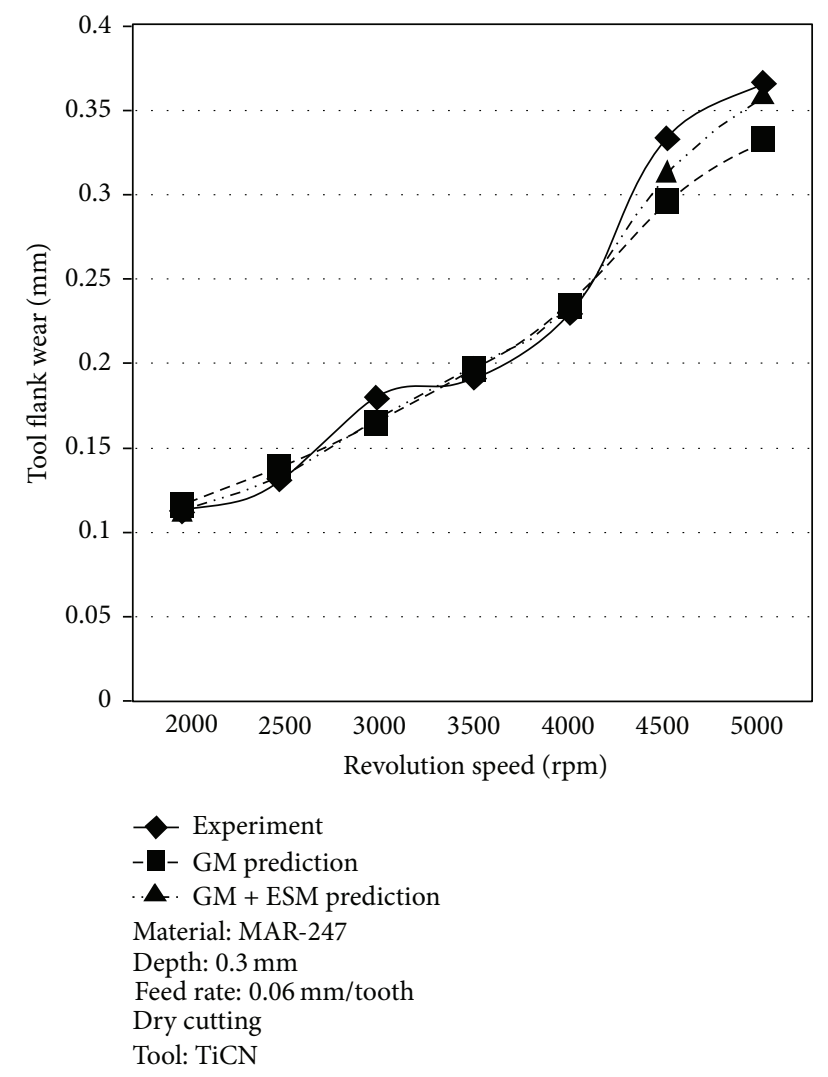

FIGURE 10: Relationships between revolution speed of main shaft and tool wear prediction.

revolution speed brings about severer tool wear and then worsens the surface roughness, regardless of the tool K10 or coated TiN and TiCN tools. In Figure 13, it shows that the improvement of feed rate of each tooth produces a relative fine surface accuracy, which is because the lower feed rate may result in a severer tool wear, so that the surface roughness is accordingly worse, as shown in Figure 8. Moreover, the coated tools have a better performance in terms of tool life and surface roughness than the uncoated tools.

Among the prediction methods, the improved model $\mathrm{GM}(1,1)+$ MSE performs better than the common model $\operatorname{GM}(1,1)$. From Figure 14, it can be learned that the error of MAPE is 0.0003 after it is improved, smaller than 0.003 , while that of RMSE is 0.0006 , smaller than 0.015 . Therefore, when the primitive experimental values are increasing progressively, the improved model $\mathrm{GM}(1,1)+$ MSE can reduce the errors.

\section{Conclusion}

The study mainly explores and predicts the tool wear and surface accuracy through applying grey prediction model in predicting the cutting characteristics of nickel-base material MAR-M247. The following conclusions are obtained based on the errors generated in different grey prediction models and after improving the model.

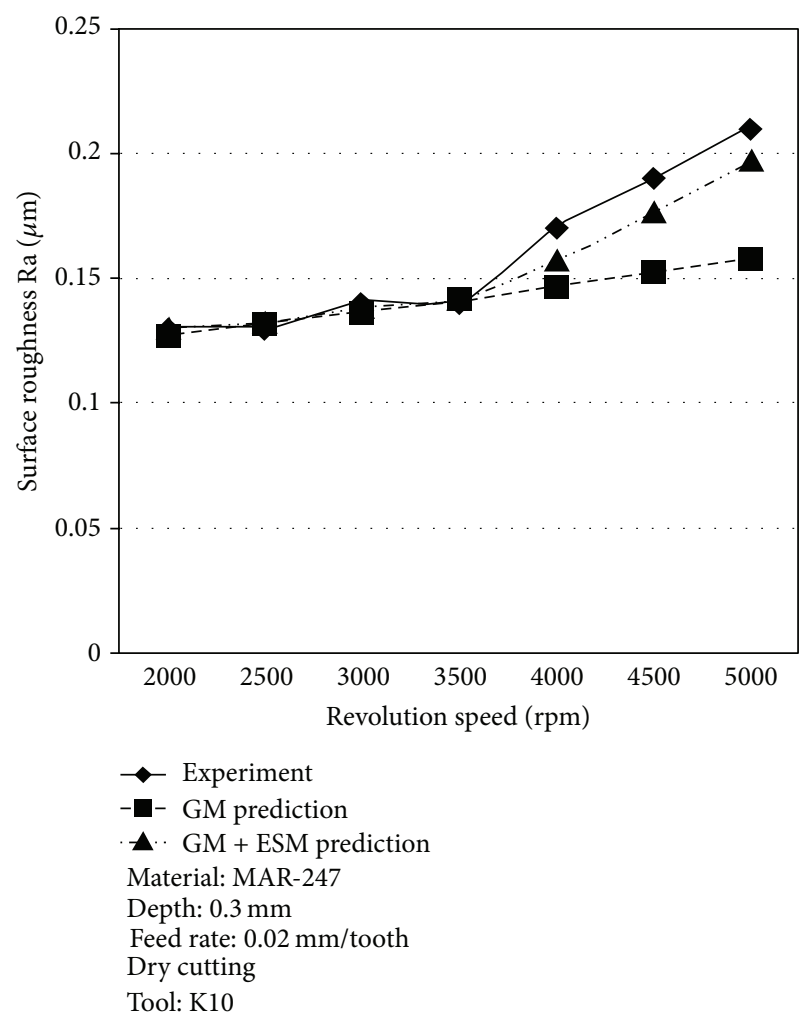

FIGURE 11: Relationships between revolution speed of main shaft and surface roughness.

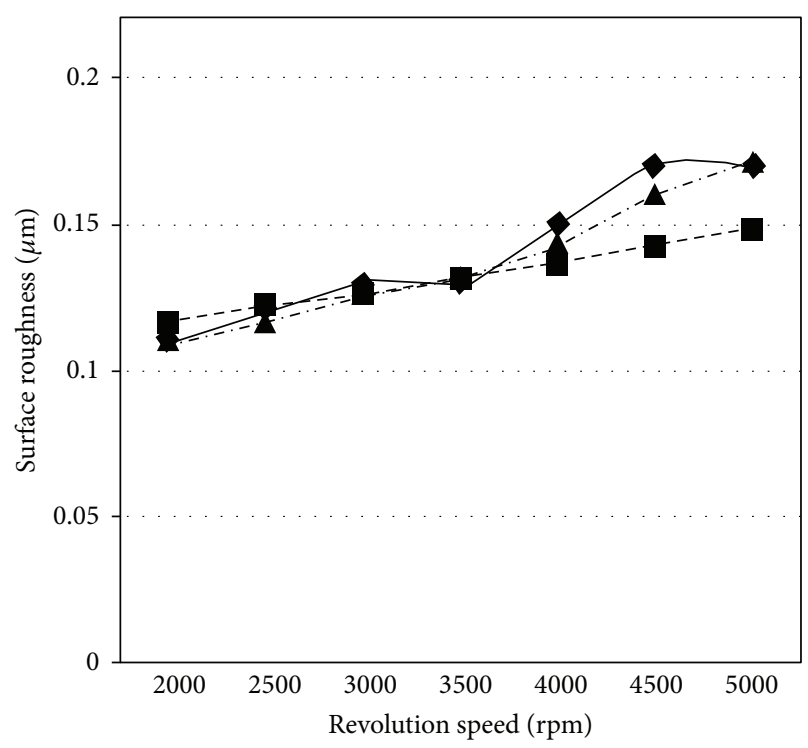

\footnotetext{
$\checkmark$ Experiment

GM prediction

- - GM + ESM prediction

Material: MAR-247

Depth: $0.3 \mathrm{~mm}$

Feed rate: $0.02 \mathrm{~mm} /$ tooth

Dry cutting

Tool: TiCN
}

FIGURE 12: Relationships between revolution speed of main shaft and surface roughness. 


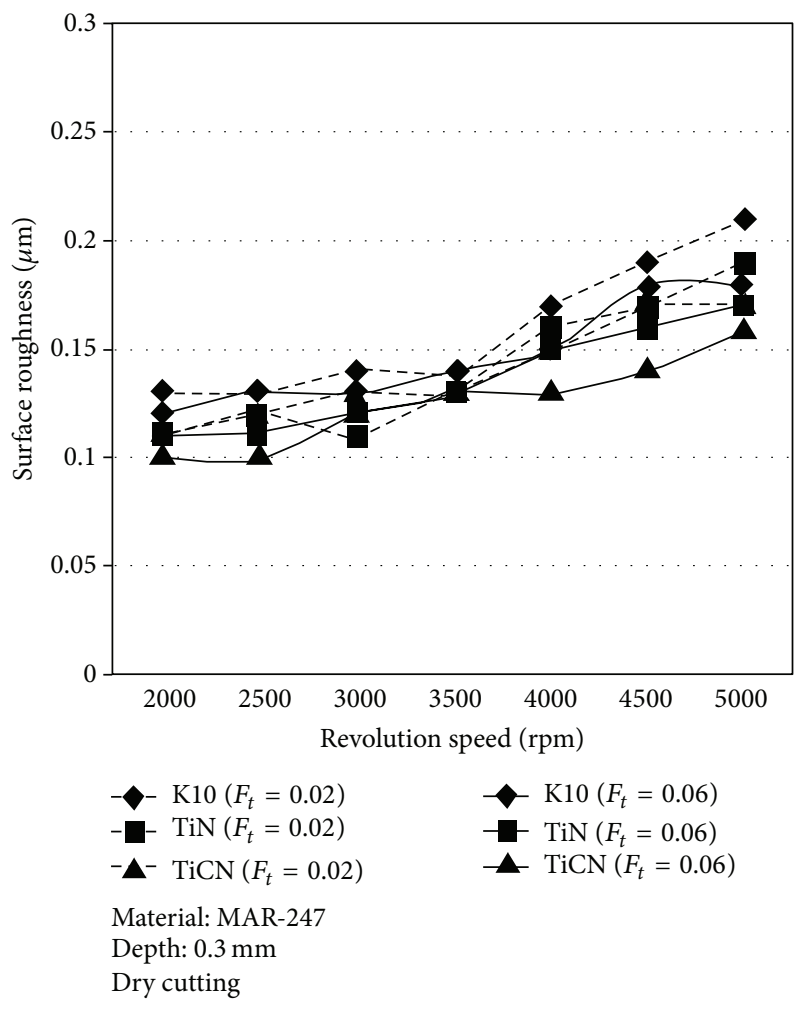

FIGURE 13: Relationships between revolutions.

(1) With the same tool materials, the higher the revolving speed is, the severer the tool wear is, which is mainly because the frictions generated in the unit time are relatively more, while the uncoated tools show much severer tool wear than the coated tools.

(2) With the same tool materials, the slower feed rate of each tooth results in a severer tool wear, for the accumulated temperature is relatively high under a low feed rate causing the precipitation hardening and material polymerization, while the uncoated tools show much severer tool wear than the coated TiN and TiCN tools.

(3) In the grey prediction model for tool wear, the improved model can significantly reduce the errors, so $\operatorname{GM}(1,1)+$ MSE is superior to $\operatorname{GM}(1,1)$.

(4) The primary factors degrading the surface roughness include the tool wear and feed rate. With the same feed rate, the surface roughness is mainly affected by the tool wear, while the surface roughness of uncoated tools is much worse than that of coated TiN and TiCN tools.

(5) In the grey prediction model for work-piece surface roughness, the errors can be notably reduced after the model is improved; therefore, $\operatorname{GM}(1,1)+$ MSE is superior to $\operatorname{GM}(1,1)$. Consequently, the divergence model can be used for analysis if the primitive experimental values are with progressive increase.

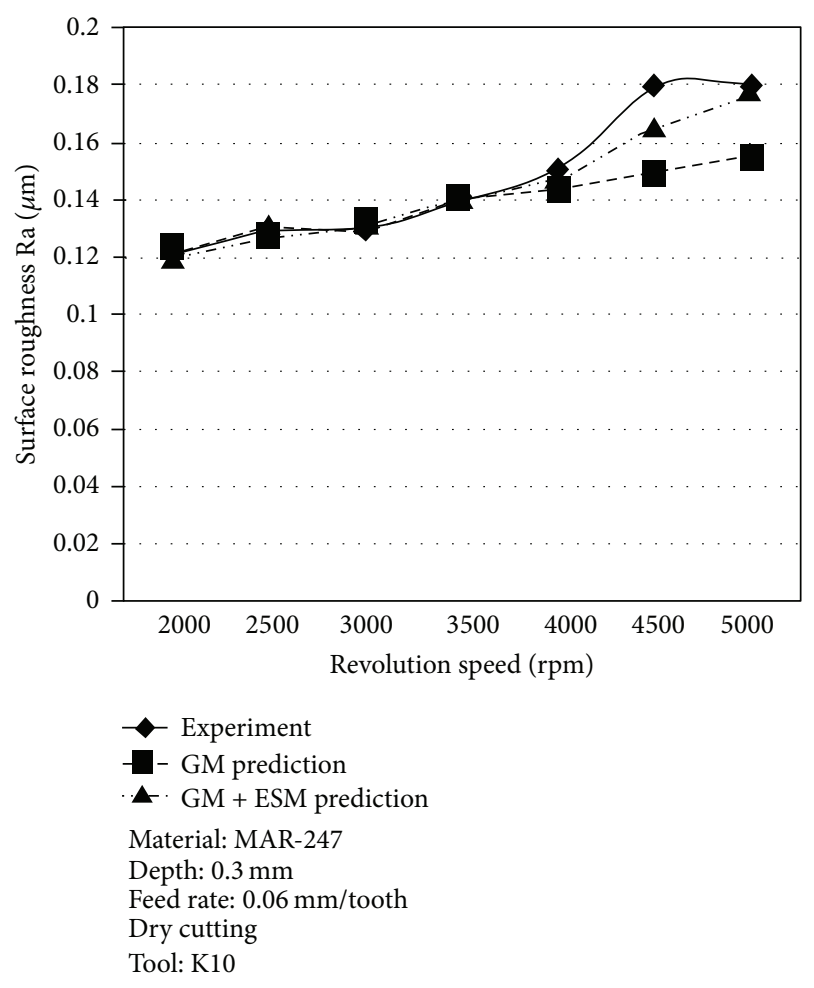

FIGURE 14: Relationships between revolution speed of main shaft and surface roughness.

\section{Conflict of Interests}

The author declares that there is no conflict of interests regarding the publication of this paper.

\section{References}

[1] D. J. Liao and C. C. Lin, The machinability of super-alloys inconel-718 and Mar-M247 with end-milling [M.S. thesis], North Carolina Central University (NCCU), Durham, NC, USA, 2001.

[2] S. L. Lin and L. L. Lin, The influence of different coated tools when milling MAR-M247 [M.S. thesis], NCCU, 2002.

[3] A. Kumar, S. Maheshwari, C. Sharma, and N. Beri, "A study of multiobjective parametric optimization of silicon abrasive mixed electrical discharge machining of tool steel," Materials and Manufacturing Processes, vol. 25, no. 10, pp. 1041-1047, 2010.

[4] N. Beri, S. Maheshwari, C. Sharma, and A. Kumar, "Multiobjective parametric optimisation during electrical discharge machining of Inconel 718 with different electrodes," International Journal of Materials Engineering Innovation, vol. 2, no. 34, pp. 236-248, 2011.

[5] C. T. Sims and W. C. Hagel, The Super-Alloys, Wiley, General Electric Company, New York, NY, USA, 1972.

[6] J. L. Deng, Grey System Theory and Application, Gau-lih Book, New Taipei City, Taiwan, 2000.

[7] J. L. Deng, "Introduction to Grey system theory," Journal of Grey System, vol. 1, no. 1, pp. 1-24, 1989.

[8] J. L. Deng and H. Gau, Grey Prediction Theory and Application, Chuan-Hwn Book, Gau-Lih Book, Taipei, Taiwan, 1996.

[9] N. Beri, A. Kumar, H. Pungotra, and G. Mittal, "Efficiency optimization during electric discharge machining of $\mathrm{H}-13$ 
steel based on grey relation analysis," International Journal of Scientific \& Engineering and Research, vol. 4, no. 8, 2013.

[10] E. Kayacan, B. Ulutas, and O. Kaynak, "Grey system theorybased models in time series prediction," Expert Systems with Applications, vol. 37, no. 2, pp. 1784-1789, 2010.

[11] C. T. Lin and L. H. Chen, "Grey analysis of bank re-decreasing the required reserve ratio," Journal of Grey System, vol. 4, no. 2, pp. 119-132, 1999.

[12] K. C. Hung and G. R. Wu, "Improvements formula GM $(1,1)$ Grey forecasting model for electricity demand forecasting Taiwan Study," Journal of Engineering Technology and Education, vol. 5, no. 3, pp. 446-458, 2008. 

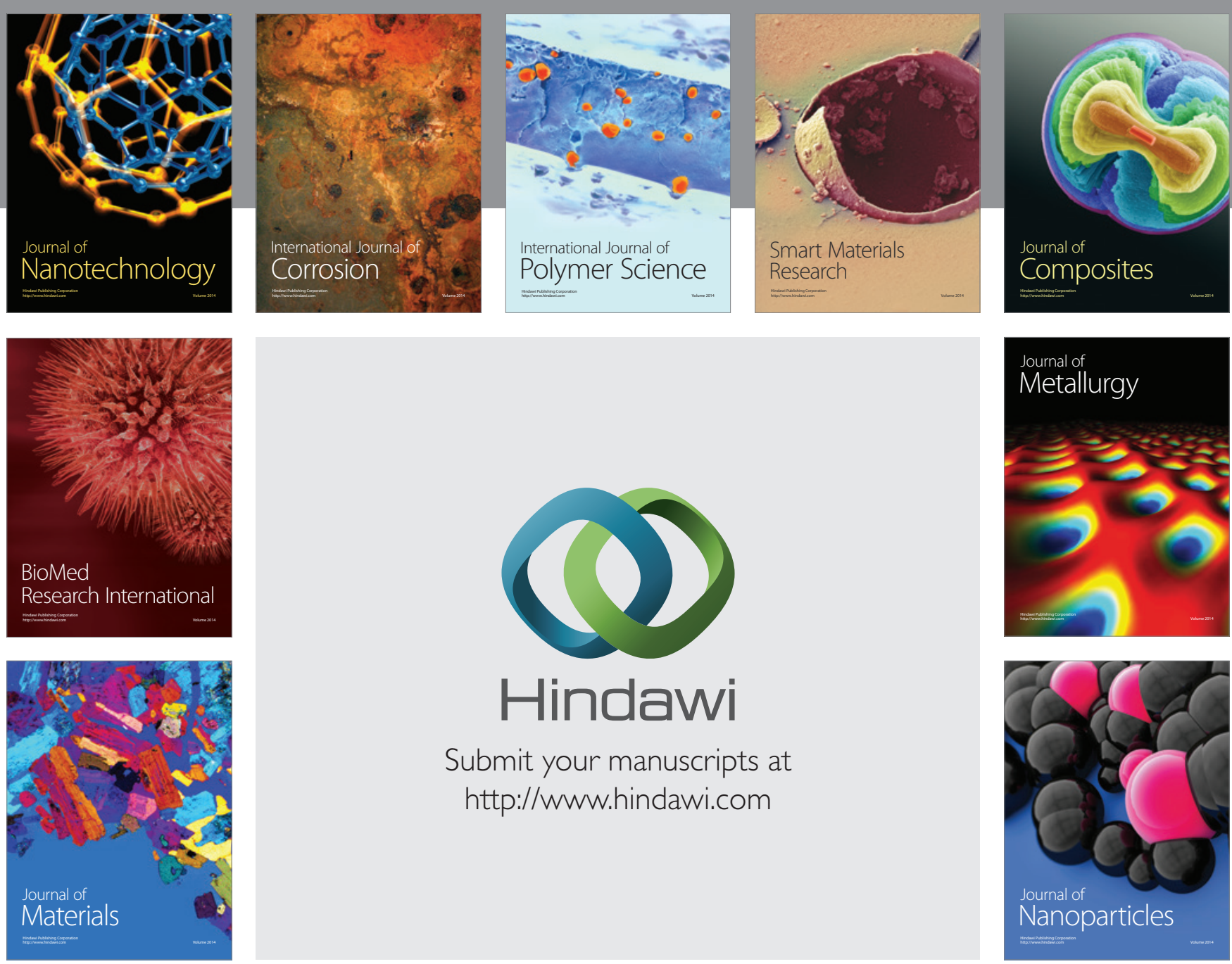

Submit your manuscripts at http://www.hindawi.com
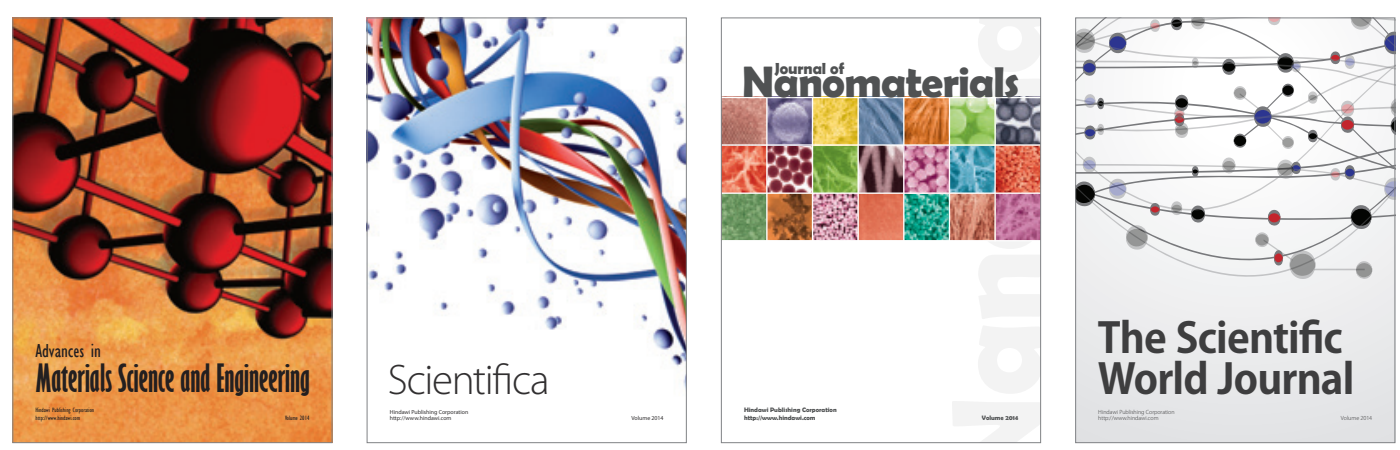

\section{The Scientific World Journal}
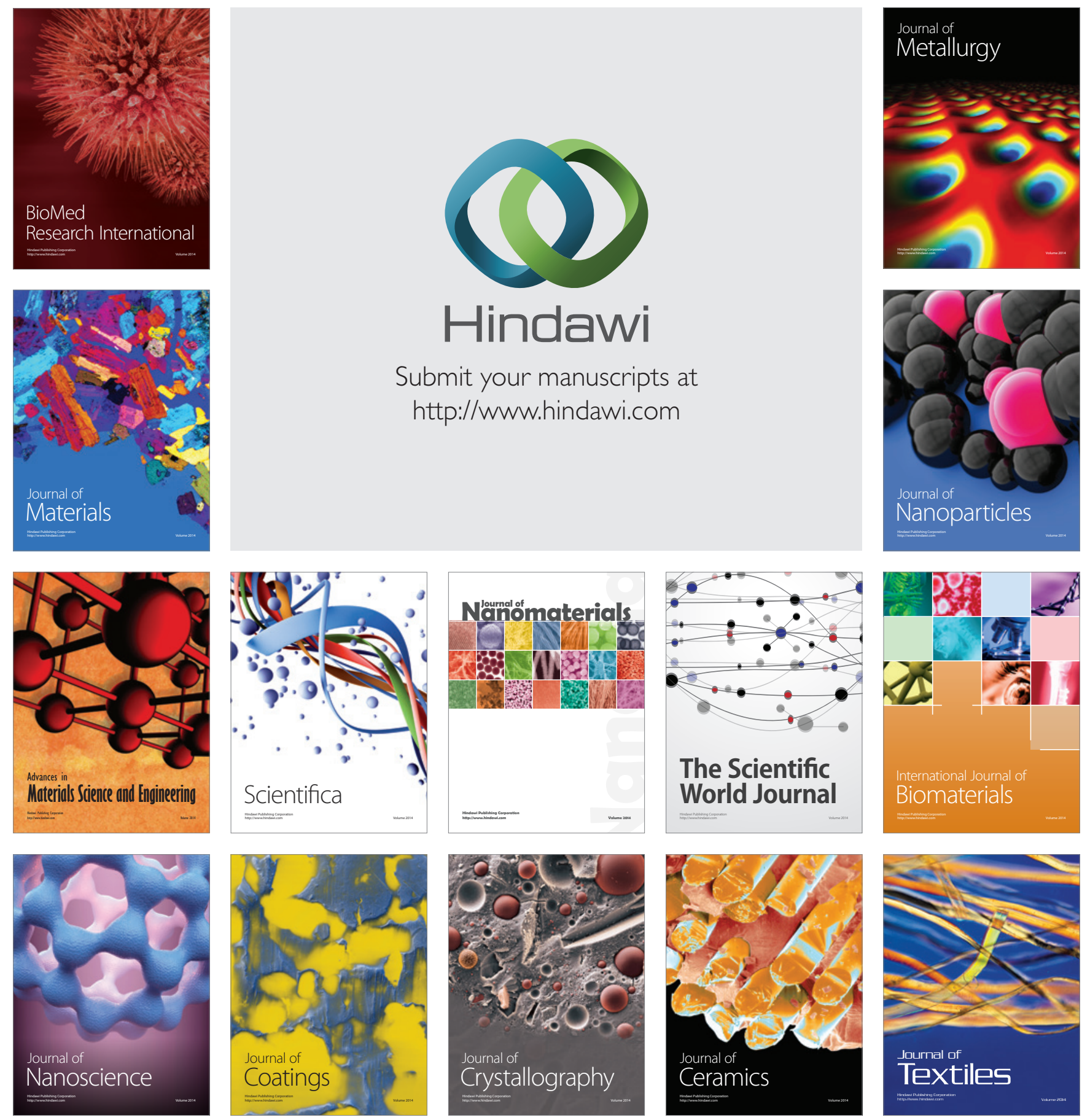